\title{
Pregabalin abuse as a sleep medication: case reports
}

\begin{abstract}
Background: Pregabalin is well known as an anxiolytic, analgesic and antiepileptic medication. It was alarming for mental health service when it started being used as addictive agent by patients for its hypnotic properties.
\end{abstract}

Methods: We report two cases from Community Mental Health Team who started abusing their prescribed Pregabalin. They were closely observed in community and on inpatient unit for their pattern and extent of drug abuse and dependence criteria.

Results: Both the patients developed dependence syndrome indicated by tolerance, craving and presented with withdrawal symptoms on stopping Pregabalin. They needed replacement medication to manage withdrawal symptoms.

Conclusion: Pregabalin has strong addictive potential and mostly abused by people for its hypnotic or sleep-inducing properties. The development of dependence and nature of withdrawal symptoms vary from person to person; however, Benzodiazepines with long half-life have good potential to manage Pregabalin withdrawal symptoms.

Keywords: pregabalin abuse, pregabalin as hypnotic, pregabalin controlled medication, sleep, anxiety, benzodiazepine, gabapentin
Volume 4 Issue 2 - 2020

\author{
Basavaraja Papanna,' Carlo Lazzari, ${ }^{2}$ Kapil \\ Kulkarni,' Sivasankar Perumal' \\ 'Essex Partnership University NHS Foundation Trust, UK \\ ${ }^{2}$ South West Yorkshire University NHS Trust, UK
}

Correspondence: Basavaraja Papanna, Essex Partnership University NHS Foundation Trust, UK, Email b.papana@nhs.net

Received: June 22, 2020 | Published: August 26, 2020

\section{Introduction}

Pregabalin is widely used in psychiatric practice to treat people with anxiety problems and neuropathic pain. It also has role as adjunctive therapy for focal or generalised seizure disorder. ${ }^{1}$ Pregabalin is a gabapentinoid which is derivative of GABA amino butyric acid. It was developed as a successor to gabapentin. ${ }^{2}$ In United Kingdom, prescription of gabapentin and pregabalin has increased by $150 \%$ and $350 \%$, respectively in the last 5 years. In addition to licensed use in neuropathic pain, both have become a tempting option for other refractory pain syndromes with or without a clear organic substrate. ${ }^{3}$ Pregabalin has also been reported effective in improving sleep quality in patients who suffer from a generalized anxiety disorder, fibromyalgia, neuralgia, or epilepsy. ${ }^{4-6}$ Multiple case reports and pharmacovigilance data have shown that Pregabalin is now widely known to induce addictive behaviours. There has been a recent increase in deaths related to Pregabalin use in the United Kingdom. This is due to Pregabalin abuse by the service users along with other substances like opioid, alcohol, to potentiate their effects. ${ }^{7-9}$ The mechanism of addictive potential can be explained by the fact that therapeutic doses of Gabapentinoids are dose dependently associated with a modest increase of the extracellular GABA-concentration in brain tissue. Thus, they have weak GABA mimetic features that most likely drive the relaxation and euphoria experienced especially in the beginning of the drug therapy and during an overdose. There is a substantial tolerance against this euphoric high which is typical for addictive GABA mimetics like benzodiazepines or Propofol. ${ }^{7}$ Pregabalin is completely absorbed when taken orally with linear doseabsorption relationship. ${ }^{10}$ It is eliminated via the kidneys with minimal metabolism and has no significant drug-drug interactions. ${ }^{10}$ The Pregabalin has a half-life of 5.5-6.7 hours, independent of dose and repeated dose administration. ${ }^{11}$ Findings from Australian studies are consistent with researches indicating the serious and increasing harms associated with Pregabalin worldwide. ${ }^{9,12,13}$ This built a rationale for regulation in Australia and similar approaches being considered in the United Kingdom..$^{14,15}$ In line with this, The UK government is to reclassify the prescription drug Pregabalin as a class $\mathrm{C}$ controlled substance, after experts issued safety warnings following an increase in deaths linked to its use. ${ }^{16} \mathrm{We}$ present here the two cases of Pregabalin misuse identified in the local community mental health team; we have highlighted a few related concerns of abuse, including intake of large dosages and use in sleep disorder.

\section{Case reports}

\section{Case I}

Miss A is a 26-year-old lady known to local mental health services with a diagnosis of Emotionally Unstable Personality Disorder, Depression and Anxiety. She is also known to have history of alcohol misuse and occasional cocaine abuse. Due to the nature of illness particularly personality issues she has had impulsive attempts of selfharm and suicide by overdose of medication, ligature, and cutting her wrists. There were several admissions to the psychiatric units both voluntarily and following detention under Mental Health Act. ${ }^{17}$ She was on regular medications: Sertraline 200mg OD, Quetiapine 200mg Nocte and Pregabalin 200mg BD along with Clonazepam 1mg up to $\mathrm{BD}$ as PRN medication. Over the treatment period, there were concerns raised regarding Mrs A about non-compliance and stockpiling the medication; hence she was started on depot medication. However, after three months, Miss A ceased engaging with the Community Care Coordinator and did not attend clinics for her ongoing regular depot injection. The major concern raised by Care Coordinator was that Miss A was stockpiling and misusing Pregabalin stating that it helps with her sleep. The Mrs. A gradually increased the dosage of Pregabalin by herself up to an alleged daily amount of $3000 \mathrm{mg}$. A multidisciplinary team meeting was held to discuss her care plan and manage her for Pregabalin abuse. When Mrs. failed to collect prescription from Community Mental Health Team, she managed to collect these high doses of Pregabalin tablets either through a new General Practitioner 
or started buying it on streets from drug peddler. Mrs. A bought Pregabalin online as well and started collecting at home. She used to abuse Pregabalin along with other illicit drugs. As per history by Mrs. A, on a few occasions, she tried to discontinue Pregabalin which resulted in very significant difficulty to with her sleep, high level of anxiety, extremely irritability and few times aggression and suicidal ideations. ${ }^{18}$ When Mrs A reported ongoing suicidal intent she was considered for inpatient admission which she agreed informally and then needed to be detained under MHA. ${ }^{17}$ She needed very close observation level 4 on the ward due to multiple suicide attempts by ligature. ${ }^{19}$ Prior to admission she was using high doses of Pregabalin and on ward she started re-experiencing Pregabalin discontinuation symptoms with major concerns with her sleep and anxiety levels. ${ }^{18}$ To treat the discontinuation symptoms she was started on Diazepam with appropriate dose titration. Her withdrawal symptoms were well managed with regular Diazepam 2mg BD along with Lorazepam up to $2 \mathrm{mg}$ daily as PRN medication. There was noticeable improvement with sleep problem along with reduction of level of anxiety, suicidal thoughts and irritability.

\section{Case 2}

Mrs B is a 54-year-old lady suffering from chronic fatigue syndrome (Fibromyalgia) and back pain for many years. She has been under pain management clinic and prescribed various pain medications including Gabapentin and Oromorph. She attended Community Mental Health Team with low mood, poor and increased anxiety about trivial things. Her mood and anxiety were triggered by her ongoing physical pain. Mrs B was prescribed Duloxetine and Zopiclone to help with her mood and sleep but unfortunately both medications showed minimal benefit. Mrs B was involved physiotherapy and hydrotherapy for pain which helped her to some extent. Over the period Mrs struggled more with generalised anxiety and she was prescribed Pregabalin, initially with $75 \mathrm{mg}$ BD and then increasing it to $150 \mathrm{mg}$ BD in few weeks' time. Mrs B noticed significant improvement with sleep and anxiety and requested to increase Pregabalin dose to $300 \mathrm{mg}$ BD. However, the medical team was reluctant to increase it as she was already on a polypharmacy which might cause over sedation and related complications. She was explained very well about the risks with increasing Pregabalin in addition to her current medication. Mrs B had capacity to understand consequence and complications of polypharmacy; however, she claimed that she was buying Pregabalin from the street as it is only medication which helps her with sleep. She found Pregabalin having very strong sedative and hypnotic effect for her. Mrs B appeared argumentative and very demanding to get it although risk of over sedation, respiratory depression associated with Pregabalin particularly using more than prescribed dose especially along with other sedative medications were explained to her. Mrs B mentioned that when she stopped Pregabalin or reduced dose for few days she started becoming unwell and had severe insomnia, flu like symptoms with severe anxiety and increased irritability. It was very clear from Mrs B's explanation that she developed dependence for Pregabalin and she was experiencing withdrawal symptoms on reducing the dose or stopping Pregabalin. Cross titration with benzodiazepine helped to manage withdrawal symptoms for Mrs B.

\section{Discussion}

In above case reports, patient Mrs A was abusing Pregabalin particularly as hypnotic to induce sleep. There was a constant demand for Pregabalin with rationale that it helps with anxiety problem but the fact was that it was being misused. Multiple researches have shown that Pregabalin discontinuation causes withdrawal symptoms similar to Benzodiazepine withdrawal with inability to sleep, excessive anxiety, tremor and restlessness. ${ }^{18}$ There have been few incidents when Pregabalin dependent patients have taken overdose with the intention to die. ${ }^{14}$ We have come across number of patients under our care in Community Mental Health Team, taking extra dose by themselves as their need to go to sleep. On detailed history during clinics, Pregabalin is often bought by many patients illegally to use as sedative and hypnotic. The first case Mrs. A described here showed that Pregabalin abusers are often individuals with a history of poly substance misuse. The high level of dosages reported with Mrs. A was in excess of daily maximum level (600mg daily) and clearly were reason of concern. Online purchase of Pregabalin was another significant concern, making it easy for service users to buy online and misuse. Thus, changing its status as a controlled drug in United Kingdom recently from 1 Aril 2019 has been a major step in preventing its abuse. Pregabalin along with Gabapentin are now reclassified as controlled drugs under Drug Misuse act 1971. ${ }^{10}$ However availability of Pregabalin with drug peddlers still remains a concern as it is with any other illicit drug.

In terms of management for Mrs A, it typically involved symptomatic treatment with long acting benzodiazepine to reduce distress with withdrawal symptoms and gradually tapering the dose over the period of time. This approach helped Miss A to come out of withdrawal symptoms. Mrs A required admission due to risk with suicidal ideations and need close monitoring. The randomized controlled study by Arnold Lelsy et al., ${ }^{16}$ reported that pregabalin improved pain in fibromyalgia patients with or without depressive or anxiety symptoms..$^{20}$ The pain relief property of Pregabalin can mediate the improvement in anxiety and depression due to reduced pain which in turns improves sleep quality. ${ }^{20}$ This applied to Mrs B who reported improvement in her sleep following increasing dose of Pregabalin. Another open label single arm study by Cho Yong Won \& Mei Ling Song ${ }^{20}$ mentioned that Pregabalin is effective in the successful discontinuation of hypnotics and it improves sleep quality in patients with insomnia. ${ }^{21}$ This explains effect experienced by Mrs B and her repeated demand for Pregabalin. It is interesting to note that in both cases, Pregabalin was started for medical reasons. However due to emergence of high tolerance and dependence, increasing dosages were eventually self-administered by patients themselves. The use of Pregabalin became a compulsion for them without which they could not function well in their daily life. It was very clear that sudden discontinuation of Pregabalin was associated with physical as well as psychological withdrawal symptoms, suggesting the high potential of Pregabalin to develop dependence. Benzodiazepines with long halflife were useful to manage withdrawal effects in both the patients.

\section{Conclusion}

The conclusion we can draw from above two cases is that Pregabalin has strong potential to develop dependence syndrome with symptoms which are mostly similar to Benzodiazepine dependence. Along with anti anxiety and analgesic properties, it has strong hypnotic properties as well and it contributes significantly towards development of dependence. Pregabalin is available on streets illegally and it is easily accessible for many patients to self-medicate increasing risks to develop addiction. In the treatment of Pregabalin dependence, Benzodiazepines with longer half-life remain first line agents. 


\section{Acknowledgments}

None.

\section{Conflicts of interest}

The authors declare that there are no conflicts of interest.

\section{Funding}

None.

\section{References}

1. Joint Formulary Committee. British National Formulary (online) London: BMJ Group and Pharmaceutical Press.

2. Kaye AD, Vadivelu N, Urman R, et al. (editors). Substance Abuse. Springer. 2014. 324p.

3. Health and social care information centre. 2015.

4. Russell IJ, Crofford LJ, Leon T, et al. The effects of pregabalin on sleep disturbance symptoms among individuals with fibromyalgia syndrome. Sleep Med. 2009;10(6):604-610.

5. Sabatowski R, Gálvez R, Cherry DA, et al. Pregabalin reduces pain and improves sleep and mood disturbances in patients with post-herpetic neuralgia: results of a randomised, placebo-controlled clinical trial. Pain. 2014;109(1-2):26-35.

6. Bazil CW, Dave J, Cole J, et al. Pregabalin increases slow-wave sleep and may improve attention in patients with partial epilepsy and insomnia. Epilepsy Behav. 2012;23(4):422-425.

7. Gahr M, Freudenmann RW, Hiemke C, et al. Pregabalin abuse and dependence in Germany: results from a database query. Eur $J$ Clin Pharmacol. 2013;69(6):1335-1342.

8. Broadening and promoting the scientific understanding of addiction.
9. Schifano F. Misuse and abuse of pregabalin and gabapentin: cause for concern?. CNS Drugs. 2014;28(6):491-496.

10. Taylor CP, Angelotti T, Fauman E. Pharmacology and mechanism of action of pregabalin: the calcium channel alpha2-delta (alpha2-delta) subunit as a target for antiepileptic drug discovery. Epilepsy Res. 2007;73(2):137-150.

11. French JA, Kugler AR, Robbins JL, et al. Dose-response trial of pregabalin adjunctive therapy in patients with partial seizures. Neurology. 2003;60(10):1631-1637.

12. Evoy KE, Morrison MD, Saklad SR. Abuse and misuse of pregabalin and gabapentin. Drugs. 2017;77(4):403-426.

13. Iacobucci G. UK government to reclassify pregabalin and gabapentin after rise in deaths. BMJ. 2017:358:j4441.

14. Bonnet U, Scherbaum N. How addictive are gabapentin and pregabalin? a systematic review. Eur Neuropsychopharmacol. 2017;27(12):1185-1215.

15. Mayor Susan. Pregabalin and gabapentin become controlled drugs to cut deaths from misuse. BMJ. 2018;363:k4364.

16. Arnold LM, Leon T, Whalen E, et al. Relationships among pain and depressive and anxiety symptoms in clinical trials of pregabalin in fibromyalgia. Psychosomatics. 2010;51(6):489-497.

17. Kettles AM, Moir E, Woods P, et al. Is there a relationship between risk assessment and observation level?. J Psychiatr Ment Health Nurs. 2004;11(2):156-164.

18. Controlled drug: pregabalin and gabapentin.

19. Naveed S, Faquih AE, Chaudhary A. Pregabalin-associated discontinuation symptoms: a case report. Cureus. 2018;10(10):e3425.

20. Cho YW, Song ML. Effects of pregabalin in patients with hypnoticdependent insomnia. J Clin Sleep Med. 2014;10(5):545-550.

21. http://www.legislation.gov.uk/ukpga/1983/20/contents 